\title{
Long-Term Follow-up of Polypropylene Bone Anchored Slings
}

\author{
Bhavin N. Patel, Emma Longo, Fred E. Govier, Kathleen C. Kobashi, Alvaro Lucioni \\ Section of Urology and Renal Transplantation, Virginia Mason Medical Center, Seattle, WA, USA
}

\section{ABSTRACT}

Introduction: The transvaginal bone anchored polypropylene sling (BAS) has proven to be a successful treatment for patients with SUI. However, there is limited data on long-term outcomes following BAS with polypropylene mesh. We report our series of patients who had at least 3 years of follow-up after placement of BAS.

Materials and Methods: A retrospective review of prospectively collected data of patients undergoing BAS for stress urinary incontinence (SUI) with minimum 3 year follow-up was performed. Outcomes and complications were determined from annual mailed post-operative questionnaires.

Results: 142 patients who had undergone BAS and had answered post-operative questionnaires at a minimum of 3 years were identified. Average follow-up was 58 months (range 36-97 months). The overall success rate was 71\% with a dry rate of $27 \%$. Complications occurred in 9\% of patients, more commonly in patients without a history of anti-incontinence procedure.

Conclusions: Although less commonly used, BAS with polypropylene mesh is associated with an acceptable success rate at long term follow-up but a low completely dry rate.

\section{ARTICLE INFO}

\author{
Key words: \\ Polypropylenes; Suburethral \\ Slings; Urinary Incontinence; \\ Body Mass Index
}

Int Braz J Urol. 2013; 39: 493-7

Submitted for publication:

February 05, 2013

Accepted after revision:

June 14, 2013

\section{INTRODUCTION}

As the data on retropubic slings has matured, transvaginal bone anchored slings (BAS) have fallen out of favor in the treatment of female stress urinary incontinence (SUI). BAS were mainly used for indications of intrinsic sphincter deficiency and in patients who had failed previous anti-incontinence procedures as a salvage procedure (1). While they are effective (2) and associated with a minimal risk of complication (2), the high cost of bone anchoring devices and reports of poor success (3) have likely contributed to the decreased usage of the BAS.

Success rates of BAS in the literature are extremely variable and range from 60.4 to $94 \%$ $(2,3)$. Unfortunately, many previous studies report conglomerate results of different sling materials with bone anchors despite evidence that the biomechanical material properties, such as strength and host durability, differ substantially (4). Furthermore, many of the reports on BAS are on sling materials that are not commonly used for pelvic surgery in the modern era, such as Dacron and Gore-Tex $(5,6)$. Finally, in many of these reports long-term follow-up is lacking.

In our practice, we have shifted away from bone anchor slings and gone toward different modalities in the treatment of SUI. This is because of the long-term data on midurethral slings (7) as well as reports of high success rates with autologous fascial slings (8). We do believe that bone anchored slings still have a role in the treatment of SUI, particularly in patients with a history of previous synthetic midurethral sling or in patients with poor quality autologous fascia. Despite the 
shift away from bone anchored slings, we continue to follow our patients treated for SUI with BAS. In this paper we present our long-term follow-up of patients with BAS using polypropylene mesh.

\section{MATERIALS AND METHODS}

We performed a cross-sectional analysis of an institutional, review board-approved, prospective database of patients with pelvic floor disorders seen at our institution. All women who underwent a BAS for SUI between 1998 and 2010 were included. These patients were evaluated pre-operatively with history, physical exam, urinalysis, cystoscopic evaluation, and video urodynamics. Women were excluded if they had less than three years of follow-up. Of note, patients undergoing concomitant surgeries for prolapse or hysterectomy and those with previous anti-incontinence procedures were not excluded. All patients underwent placement of a BAS with soft polypropylene mesh (Ethicon, Somerville, NJ). The procedure was done in the operating room under general or regional anesthesia and the technique has been previously described by our group (9).

Questionnaires were used to assess patients at baseline and post-operatively. At the time of their initial visit patients were given a packet of preoperative questionnaires. This included the validated Urogenital Distress Inventory - Short Form (UDI-6) and the Incontinence Impact Questionnaire - Short Form (IIQ-7). Additionally, the Virginia Mason Incontinence Questionnaire (VMIQ) was included. The VMIQ is a non-validated questionnaire reviewing the following domains: type of incontinence, severity of incontinence, presence of pelvic pain, and fecal symptoms. Postoperatively, the patients were given the same packet of questionnaires, UDI-6, IIQ-7, VMIQ, as well as an additional questionnaire to evaluate change in symptoms, their satisfaction with the surgery, complications from surgery, and need for further surgeries.

Success was defined as less than one incontinent episode per week or a greater than $70 \%$ patient perceived improvement from baseline. Dry was defined as patient response on questionnaire. Descriptive statistics, Student's t-tests, and chi- -squared analysis were performed as appropriate. $P$ values less than 0.05 were considered significant. Odds ratios were also calculated.

\section{RESULTS}

A total of 325 patients underwent a BAS during the study period. Of these patients $105 \mathrm{did}$ not provide consent to be followed with questionnaires for study purposes. Of the remaining 220 patient, 142 (65\%) met our study inclusion criteria (Table-1). The average follow-up was 58 months with a range of 36 to 97 months. Overall, the mean age of this group of patients was 64 years. The mean Valsalva leak point pressure (VLPP) was $47.7 \mathrm{~cm}$ of water, with a standard deviation

Table 1 - Overall Patient Demographics and Surgical Outcomes.

\begin{tabular}{lc}
\hline Total Patients (N) & 142 \\
\hline Average Age & $64.2 \pm 10.1$ \\
Mean Gravidity & $2.4 \pm 0.8$ \\
Mean Parity & $2.4 \pm 0.8$ \\
Previous Anti-Incontinence Surgery & $104(73 \%)$ \\
Mean BMI & $28.3 \pm 4.4$ \\
Preoperative Urgency & $9(6 \%)$ \\
VLPP & $47.7 \pm 25.5$ \\
Concomitant Surgery & $75(53 \%)$ \\
Follow-Up Range (months) & $36-97$ \\
Mean Follow-Up (months) & $101(71 \%)$ \\
Success & $57.7 \pm 16.0$ \\
\hline & \\
Lreater than 70\% improvement & $(60 \%)$ \\
\hline
\end{tabular}


of $25.5 \mathrm{~cm}$ of water. The mean body mass index (BMI) was 28.3 with a standard deviation of 4.4 and 104 (73\%) patients had undergone at least one previous procedure for SUI. The success rate, defined as less than one incontinent episode during cough, sneeze, or physical activity per week or greater than 70\% improvement from the baseline, was determined to be $71 \%$ from the most recent follow-up questionnaire. The overall dry rate was $27 \%$. There were no statistically significant differences in age, gravidity, parity, BMI, VLPP, or history of previous surgeries between patients with successful procedures and patients with failed or non-successful procedures.

BAS-related complications occurred in 13 out of $142(9.15 \%)$ patients (Table-2) and included 3 cases of transient retention (less than 4 weeks), 5 vaginal mesh extrusions, 2 prolene suture vaginal extrusions, 1 case of bladder erosion of the prolene suture, and 2 cases of persistent SUI requiring a subsequent procedure (within 6 months). Of the 5 vaginal mesh extrusions, 4 required transvaginal operative excision. One mesh extrusion was successfully treated with vaginal estrogen therapy. The case of prolene suture bladder erosion required a mini-laparotomy for repair. There were no cases of osteomyelitis or bone pain after the BAS.

When comparing those patients who had undergone a previous anti-incontinence surgery to those who had not, we found no difference in age, BMI, VLPP, gravidity, parity, success, dry rate, or follow-up. Patients without history of anti-incontinence surgery were more likely to have a sling-related complication than those with a history of a previous anti-incontinence surgery ( $p=$ 0.044 , Table-3).

\section{DISCUSSION}

Many different surgical procedures have been used in the treatment of SUI over the last few decades, including BAS. Current practice has moved away from the BAS procedure due to the risk of osteomyelitis, the high cost of the bone anchoring device, and the higher rates of mesh exposure as compared to mid-urethral slings. Despite this trend, many patients have previously undergone this surgery and their long-term outcome in regards to efficacy and complications is still poorly defined. Here we demonstrate an overall success rate of $71 \%$ at a mean follow-up of 58 months in 142 patients who underwent a BAS procedure with off-the-shelf prolene mesh. The overall dry rate was much lower at $27 \%$. Using the same definition, we previously reported a success rate of $73 \%$ and a dry rate of 30\% at one year of follow-up (9). The similar success and dry rates at 1 year and 5 years of follow-up indicates a very durable effect of BAS on symptoms. Furthermore, the long-term success rates after BAS are comparable to those reported for midurethral slings (10).

In reviewing our long-term data, we were unable to identify any patient factors, including age, body mass index, presence of urinary urge or urgency incontinence, or Valsalva leak point pressure, that were associated with outcome.

Table 2 - All complications reported in patients that underwent BAS surgery.

\begin{tabular}{lcc}
\hline Complication & N \\
\hline Transient Retention & 3 & $<3.5$ wks CIC \\
Bladder Erosion of Prolene Suture & 1 & ex-lap \\
Vaginal Mesh Extrusion & 5 & 4 had operative excision \\
Vaginal Suture Extrusion & 2 & both managed with local excision \\
Persistent SUI Requiring Surgery $(<6$ months $)$ & 2 & 1 collagen and 1 repeat BAS
\end{tabular}


Table 3 - Complications following BAS surgery in patients with and without a history of previous anti-incontinence surgery.

\begin{tabular}{lcc}
\hline Complication & $\begin{array}{c}\text { Previous Anti-Incontinence } \\
\text { Procedure } \\
(\mathrm{N}=104)\end{array}$ & $\begin{array}{c}\text { No Previous Anti-Incontinence } \\
\text { Procedure } \\
(\mathrm{N}=39)\end{array}$ \\
\hline Temporary Retention $(<4$ weeks) & $1(1 \%)$ & $2(5 \%)$ \\
Bladder Erosion of Suture & $1(1 \%)$ & $0(0 \%)$ \\
Vaginal Mesh Exposure & $2(2 \%)$ & $3(8 \%)$ \\
Vaginal Suture Exposure & $1(1 \%)$ & $1(3 \%)$ \\
Persistent SUl (subsequent procedure within 6 months) & $1(1 \%)$ & $1(3 \%)$ \\
\hline Total complications & $6(6 \%)$ & $7(18 \%)$ \\
\hline
\end{tabular}

However, patients with poor surgical outcomes were on average 5 years older than patients with successful outcomes. Although not statistically significant $(\mathrm{p}=0.2)$, this trend is supported in the sling literature, with reports of lower success rates in older patients (11).

The overall complication rate in this cohort of patients was $9.1 \%$, with a rate of vaginal exposure / extrusion of 3.5\% or 5 of 142 patients. The total number of complications and vaginal exposures reported here is likely lower than the true number because we excluded all patients with less than 3 years of follow-up and follow-up questionnaires are unlikely to capture patients with asymptomatic mesh exposure. Of note, there were no cases of osteomyelitis, which is an infrequent, but debilitating, potential complication of this surgery (12). Furthermore, patients were significantly more likely to have complications if this was their first anti-incontinence surgery.

While it is not entirely clear why we found that patients with no history of previous anti-incontinence surgery had a higher rate of complications, it is likely due to several factors. As a referral center it is uncommon for our practice to treat uncomplicated patients. Most of our patients have undergone previous anti-incontinence procedures and those patients that have not often display co-morbidities or disease states that make undergoing operative procedures potentially more risky, such as morbid obesity and a history of pelvic radiation. Therefore, patients seen at our practice that have no history of previous anti-incontinence surgery may be more likely to have confounding risk factors, possibly contributing to the increased prevalence of complications. We also speculate that the lower rate of complications, such as mesh extrusion, in patients with previous surgeries may be attributed to protection produced by scar tissue or thicker vaginal flaps due to previous surgeries.

One weakness of this paper and the literature on SUI is that it is difficult to compare definitions of success among different reports on urinary incontinence. There is no consensus in the field on how to define success after an anti-incontinence procedure. While we have been consistent with our definition of success across all publications from out institution, there are a myriad of definitions for success in the treatment of SUI and the success rate is complicated by the fact that it is completely determined by the patient. For example, a patient may be too embarrassed to admit that their surgery was unsuccessful, or a patient may not report small amounts of leakage. These factors prohibit effective comparisons among different groups analyzing the same treatment for the same disease. Another limitation of our paper is our follow-up rate. Of all patients who consented to follow-up, we had $65 \%$ of patients complete a minimum of 3 year follow-up. The outcome of 
patients lost to long-term follow-up is unknown. Another reason for patient loss is the inherent difficulty with mailed questionnaires, the fact that patients may not return them. An option would be to schedule all patients for follow-up in clinic, but this is not always medically necessary and is an unfair burden on the patient's time and resources. All this being said, a 65\% response rate is in line with the other questionnaire based studies (13-15).

An important strength of our study, when compared to others on BAS that often include multiple types of sling materials, is that we have assessed the durability, success, and outcomes of only polypropylene mesh slings. The data presented here is not an aggregate of different materials with different properties. Another strength is that we report long-term outcomes for this specific anti-incontinence procedure, data that is lacking in the literature.

\section{CONCLUSIONS}

Our study, which followed 142 patients for an average of 58 months, reveals a reasonable success rate for BAS. However, the cost of the bone anchoring device, better results with autologous fascial sling, and available long-term results from retropubic sling have limited the role of BAS in the treatment of SUI.

\section{CONFLICT OF INTEREST}

None declared.
4. Choe JM, Kothandapani R, James L, Bowling D: Autologous cadaveric, and synthetic materials used in sling surgery: comparative biomechanical analysis. Urology. 2001; 58: 482-6.

5. Madjar S, Beyar M, Nativ 0: Transvaginal bone anchored sling. Urology. 2000; 55: 422-6.

6. Choe JM, Staskin DR: Gore-Tex patch sling: 7 years later. Urology. 1999; 54: 641-6.

7. Krlin RM, Murphy AM, Ingber MS, Vasavada SP: Mid-urethral slings in female incontinence: Current status. Indian J Urol. 2011; 27: 320-5.

8. Howden NS, Zyczynski HM, Moalli PA, Sagan ER, Meyn LA, Weber AM: Comparison of autologous rectus fascia and cadaveric fascia in pubovaginal sling continence outcomes. Am J Obstet Gynecol. 2006; 194: 1444-9.

9. Rapp DE, Kobashi KC, Govier FE: Placement of a transvaginal bone-anchored sling A new minimally invasive technique is useful in stress urinaryincontinence. Am J Obstet Gynecol. 2009; 200: 345.e1-3.

10. Rapp DE, Govier FE, Kobashi KC: Outcomes following midurethral sling placement in patients with intrinsic sphincteric deficiency: comparison of Sparc and Monarc slings. Int Braz J Urol. 2009; 35: 68-75; discussion 75.

11. Kim J, Lucioni A, Govier F, Kobashi K: Worse long-term surgical outcomes in elderly patients undergoing SPARC retropubic midurethral sling placement. BJU Int. 2011; 108: 708-12.

12. Goldberg RP, Tchetgen MB, Sand PK, Koduri S, Rackley R, Appell $\mathrm{R}$, et al.: Incidence of pubic osteomyelitis after bladder neck suspension using bone anchors. Urology. 2004; 63: 704-8.

13. Svihra J, Luptak J, Svihrova V, Mesko D: Gender-specific external barriers to seeking care for urinary incontinence. Patient Prefer Adherence. 2012; 6: 773-9.

14. Wehrberger C, Madersbacher S, Jungwirth S, Fischer P, Tragl $\mathrm{KH}$ : Lower urinary tract symptoms and urinary incontinence in a geriatric cohort - a population-based analysis. BJU Int 2012; 110: 1516-21.

15. Nazemi TM, Rapp DE, Govier FE, Kobashi KC: Cadaveric fascial sling with bone anchors: minimum of 24 months of follow-up. Urology. 2008; 71: 834-8.

\section{REFERENCES}

1. Kovac SR, Cruikshank SH: Pubic bone suburethral stabilization sling for recurrent urinary incontinence. Obstet Gynecol. 1997; 89: 624-7.

2. Lucioni A, Kobashi KC: Bone-anchored suburethral sling: surgical technique and outcomes. Curr Urol Rep. 2009; 10: 384-9.

3. Carbone JM, Kavaler E, Hu JC, Raz S: Pubovaginal sling using cadaveric fascia and bone anchors: disappointing early results. J Urol. 2001; 165: 1605-11. 